\title{
Chemotherapy and Radiotherapy as the Sole Treatment of Carcinoma Breast- A Case Report
}

\author{
Yuvraj* and Prasad SN \\ J.K. Cancer Institute, India
}

Submission: February 03, 2017; Published: February 21, 2017

*Correspondence Address: Yuvraj, J.K. Cancer Institute, Kanpur, Tel no: 9792928529; Email: dryuvrajbrd@gmail.com

\section{Introduction}

Breast cancer is the most common type of cancer in women and the second leading cause of cancer related deaths, next to lung cancer. If eight women live to the age of 85 , at least one of them will develop breast cancer in her lifetime. In India as per National Cancer Registry Programme year 2012, about 144,937 women were newly detected with breast cancer of which 70, 218 women died of the disease (Figures 1 \& 2).

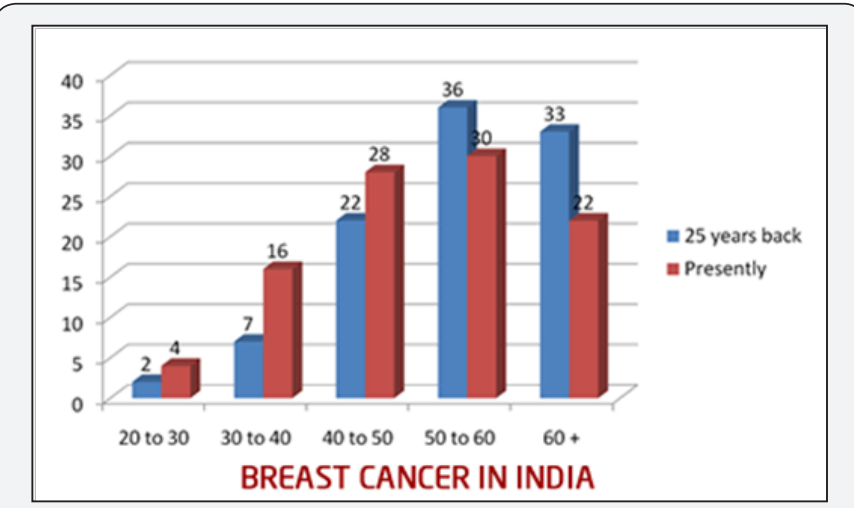

Figure 1: Breast cancer in India.

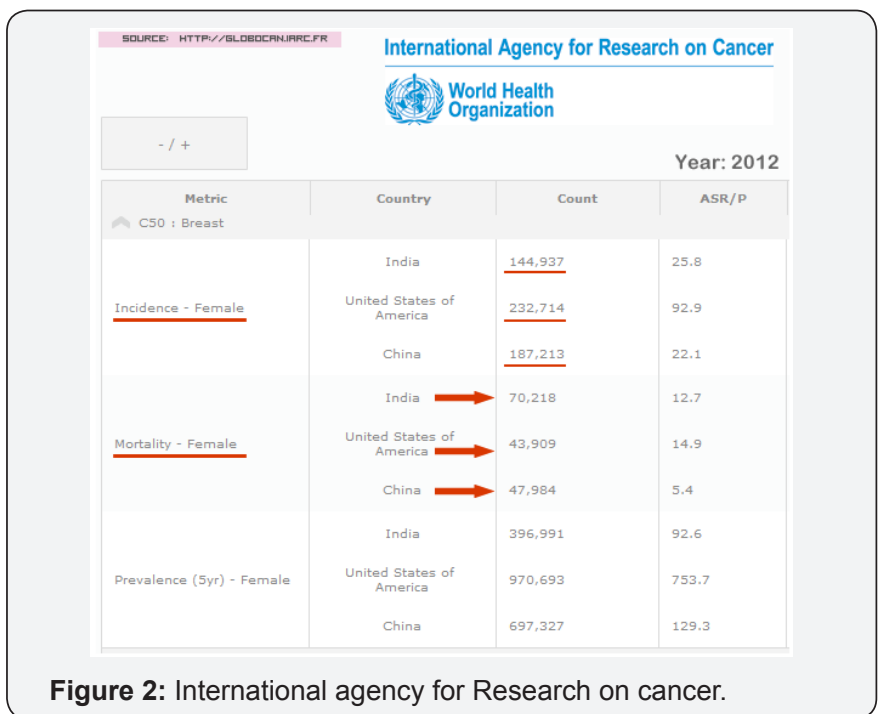

a.For a complete and optimal therapy for breast cancer, a multidisciplinary approach with input from the patient, the surgeon, the radiation oncologist, the medical oncologist, the diagnostic radiologist, the pathologist, the general practitioner, nurses, and other health professionals is needed.

b.The primary goal in the treatment of breast cancer is to control the disease with the aim of achieving cure. The other desirable outcomes of treatment include: to improve survival, minimize the risk of distant metastases and / or local recurrence, cosmesis, relief of symptoms, and the return to a better quality of life as close as possible to the life before diagnosis.

Surgery either BCS or radical followed by chemo and radiation with or without hormone therapy has been the mainstay in the treatment of carcinoma breast.

\section{Case Presentation}

A 54 yr old Indian female registered at JKCI on 5/10/2009 presented with lump in right breast since 1 month. Lump was gradually progressive, associated with slight pain. 0/E: Right breast- $6 \mathrm{X} 4 \mathrm{cms}$, firm, mobile, tender lump, was palpable over Right breast in upper inner quadrant. Nipple areola complex normal A $3 \mathrm{X} 3 \mathrm{cms}$ firm, mobile, non tender, Right axillary L.N. palpable over Right axilla.

i. Left breast and axilla- NAD. Clinically staged as cT3N1M0.

ii. Her work up included FNAC Right breast- s/o duct adeno carcinoma.

iii. USG W/A- 24/8/09(LLRH, Dept. of radio diagnosis) revealed mild hepatomegally with mild fatty liver.

iv. Left renal cyst.

v. 2D ECHO- 7/10/09 (LPS INSTITUTE) - EF 60\%. Rest NAD.

vi. $\quad$ CBC/LFT/KFT- WNL. 


\section{Cancer Therapy \& Oncology International Journal}

After complete clinical, radiological and biochemical evaluation, pt was advised surgical opinion but patient refused surgery. She was counselled multiple times thereafter as per standard protocol for the management of carcinoma breast. Despite best efforts she denied surgery. She was planned for neoadjuvant chemotherapy. She received 4 cycles of NACT (Inj. DOXORUBICIN 80mg + Inj. CYCLOPHOSPHAMIDE 800mg on d1) last cycle on 26/12/2009. On reevaluation Right breast- a 1x1 cm lump, non-tender, firm was palpable over upper inner quadrant. Right axillary L.N. $0.5 \times 0.5 \mathrm{~cm}$ was palpable. Left breast and axilla NAD. She was again advised surgical opinion but patient again refused for surgery.

She was further planned for 5 field conventional External Beam Radiotherapy EBRT/CO60/50Gy/25\#/5WEEKS/5F given b/w $16 / 2 / 2010$ to $29 / 3 / 2010$. On revaluation on $5 / 5 / 10$ - no lump was palpable over right breast and axilla. Left breast and axilla- NAD. USG B/L breasts and axilla-NAD, USG W/A- NAD, $\mathrm{X}$-RAY CHEST P/A-NAD. She was again advised surgical opinion but surgeon on call refused for surgery as there was no evidence of disease. Seeking this she was advised Tab. LETROZOLE $2.5 \mathrm{mg}$ O.D. She was evaluated every month for 6 months followed by 2 monthly till December 2010. Afterwards she was followed every 6 monthly by examining clinically, radiologically and biochemically, till this date. Her current status on $5 / 2 / 2014$ revealed $\mathrm{B} / \mathrm{L}$ breast and axilla clear with no evidence of disease.

\section{Results}

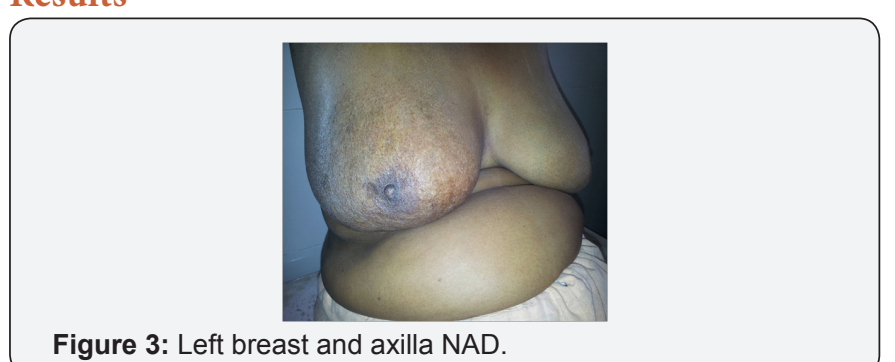

Figure 3: Left breast and axilla NAD.

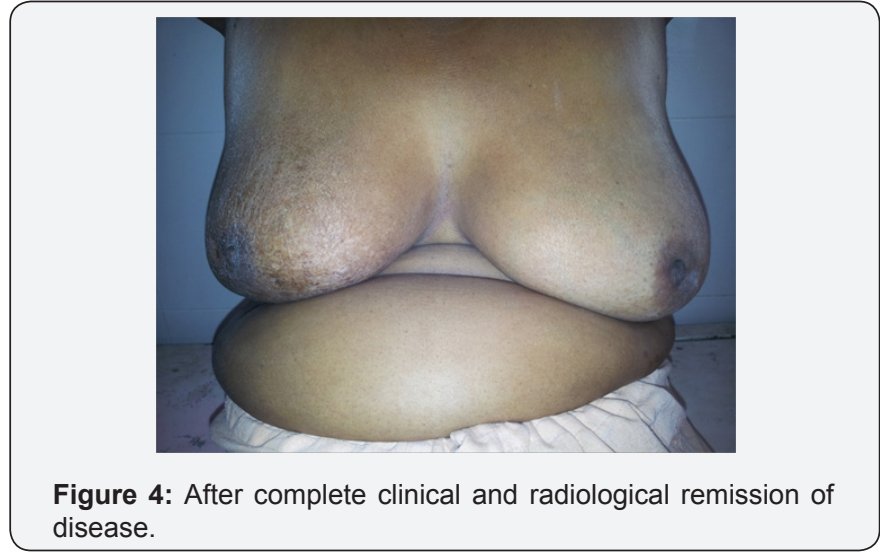

(Figures 3 \& 4) After complete clinical and radiological remission of disease with NACT and radiotherapy, pt was put on Hormone Therapy (Tab. Letrozole). She was regularly followed up and her status remains disease free to this date.

\section{Conclusion}

Taking into account more than hundred probabilities of breast cancer subtypes, a strict Knife based management protocol is always not an answer. This case suggested the possibility of a randomized study to look into the factors predictive of those subsets of patients which can be managed solely by radiation and chemotherapy particularly in an Indian scenario where by surgery is often deferred by the patient, in fear of knife induced altered body image, eventually developing anxiety, low self esteem and depression.

\section{Acknowledgement}

It is my privilege to acknowledge my sincere gratitude to $\mathrm{Dr}$ S N Prasad, Prof., J.K.C.I. Kanpur for extending his support and guidance. 